\title{
A Fuzzy Multilevel Mathematical Programming
}

\author{
M. Abdel-Aaty Maaty \\ Faculty of Science, Cairo University, Fayuom Branch, Egypt.
}

Keyworkks:

Multilevel programming, Fuzzy programming

\begin{abstract}
:
This paper gives an overview on a technique for solving single objective or multiobjective mathematical programming with fuzzy parameters in the objective functions, under the presentation of multi- decision makers in different hierarchy level (decentralized decision making situation); where the bigher level in the hierarchy can only influence aprather than dictate the choices of the lower level.
\end{abstract}

Manuscript received from $\mathrm{Dr}$.M.Abd EL-Aaty Maaty Accepted on : 1611/2001

Engineering Research Journal Vol 24,No 2, 2001 Minufiya University, Faculty Of Engineering, Shebien El-Kom, Egypt, ISSN 1110-1180. 


\section{1- Introduction}

The use of mathematical programming in decision making has a long been restricted to problems with either single overriding objective, or a variety of objective dealt with striving toward all of them simultaneously. All objectives are assumed to be those of single decision maker (DM) who has control overall decision variables [6], [8].

The single- level can be visualized as a centralized decision making system where the hgihest level in the hierarchy (headquarters, top management, or government) has enough power to dictate the decision and have them executed by lower levels (subordinates, subunite or individuals).

A more realistic formulation, however, would recognize the role of lower level as a part of the decision proccess rather than confining them within the execution of the orders of their superiors. A multilevel linear programming (MLP), a nested optimization problem, emerged as the appropriate model to formulate such decentralized decisio making where the higher level in the hierarchy take into account the reaction of the lower level decision makers when the impact of their decision is too important to be ignored [7].

This paper also, presents the concept that use the fuzzy set theory [1], [2], [4], [5] to solve such problem in order that the obtained results from using mathematical programming techniques could be used with a higher degree of confidence.

\section{2- Problem Formulation:}

A fuzzy multilevel linear programming (FMLP) is similar to standard fuzzy linear programming (FLP), except that the constraint region is modified by including a defined linear objective function; it is a nested optimization model involuing multiple $n$ problems the first is the upper one and the $n$th is the lower one [7]. The general form of (FMLP) can be defined as

EMLP (1): 
$\max _{x_{1}} f_{1}\left(x_{1}, x_{2}, \ldots, x_{n}\right)=\widetilde{c}_{1}^{(3)} x_{1}+\widetilde{c}_{2}^{(1)} x_{2}+\ldots .+\widetilde{c}_{n}^{(1)} x_{n}$, where $\quad x_{2} \quad$ solves

$\max _{\mathrm{x}_{2}} \mathrm{f}_{2}\left(\mathrm{x}_{1}, \mathrm{x}_{2}, \ldots, \mathrm{x}_{n}\right)=\widetilde{\mathrm{c}}_{1}^{(2)} \mathrm{x}_{1}+\widetilde{\mathrm{c}}^{(2)} \mathrm{x}_{2}+\ldots .+\widetilde{\mathrm{c}}_{n}^{(2)} \mathrm{x}_{n}{ }^{\prime}$

where $\quad x_{n-1}$ solves

$\max _{X_{n-1}} f_{n-1}\left(x_{1}, x_{2}, \ldots, x_{n}\right)=\widetilde{c}_{1}^{(n-1)} x_{1}+\widetilde{c}_{2}^{(n-1)} x_{2}+\ldots \ldots+\widetilde{c}_{n}^{(n-1)} x_{n}$

where $\quad x_{n}$ solves

$\max _{n} f_{n}\left(x_{1}, x_{2}, \ldots, x_{n}\right)=\widetilde{c}_{1}^{(n)} x_{1}+\widetilde{c}_{2}^{(n)} x_{2}+\ldots .+\widetilde{c}_{n}^{(n)} x_{n}$

subject to

$\mathrm{G}_{\mathrm{i}}\left(\mathrm{x}_{1}, \mathrm{x}_{2}, \ldots ., \mathrm{x}_{\mathrm{n}}\right) \leq \mathrm{b}$,

$x_{1}, x_{2}, \ldots, x_{n} \geq 0$

where $\widetilde{\mathrm{c}}_{i}^{(1)}, \widetilde{\mathrm{c}}_{i}^{(2)}, \ldots, \widetilde{\mathrm{c}}_{\mathrm{j}}^{(n)}, \mathrm{i}=1,2, \ldots, \mathrm{n}$ are a vector of fuzzy parameters and $b$ is a constant vector; $G_{i}\left(x_{i}, x_{2}, \ldots . ., x_{n}\right) i=1,2, \ldots, n$ are constraints functions; $\left(x_{1}, x_{2}, \ldots ., x_{n}\right)$ are vectors of decision variables for each level; $f_{l}, f_{2}, \ldots, f_{n}$ are the objective fun ${ }^{-\cdot} \quad$ Jnding to each decision maker (level) respectively (the un ...ective is often referred to as the objective of FMLP problem, while the lower objective is considered as just a constraints). Formulation FMLP visualizes an organizational hierarchy in which n decision makes have to improve their strategies organizational hierarchy in which $\mathrm{n}$ decision makes have to improve their strategies from jointly dependent set

$$
S=\left\{\left(x_{i}\right) \mid A_{i} x_{i} \leq b, x_{i} \geq 0,1=1,2, \ldots, n\right\} .
$$

The first decision maker who has control over $x_{1}$ make his decision first, then fixing $x_{1}=x_{1}{ }^{0}$ before the second DM select $x_{2}$, and then the resulting problem for the second DM is:

FMLP (2):

$\lim _{x_{2}} f_{2}\left(x_{1}^{0}, x_{2}, \ldots, x_{n}\right)=\widetilde{c}_{1}^{(2)} x_{1}^{0}+\widetilde{c}_{2}^{(2)} x_{2}+\ldots .+\widetilde{c}_{n}^{(2)} x_{n}$ where $\quad x_{n-1}$ solves 
$\max _{X_{n-1}} f_{n-1}\left(x_{1}^{0}, x_{2}, \ldots, x_{n}\right)=\widetilde{c}_{1}^{(n-1)} x_{1}^{0}+\widetilde{c}_{2}^{(n-1)} x_{2}+\ldots . .+\widetilde{c}_{n}^{(n-1)} x_{n}$

where $\quad x_{n} \quad$ solves

$\max _{X_{1}} f_{n}\left(x_{1}^{0}, x_{2}, \ldots, x_{n}\right)=\widetilde{c}_{1}^{(n)} x_{1}^{0}+\widetilde{c}_{2}^{(n)} x_{2}+\ldots \ldots+\widetilde{c}_{n}^{(n)} x_{n}$

subject to

$G_{i}\left(x_{1}{ }^{0}, x_{2}, \ldots, x_{n}\right) \leq b$,

$x_{1}{ }^{0}, x_{2}, \ldots, x_{n} \geq 0$

and then fixing $x_{2}=x_{2}{ }^{0}$ bfore the third DM select $x_{3}$ and so on to the $n$th decision maker.

\section{Definltion 2.1: (memborshlp)}

A real fuzzy number $\widetilde{P}$ is a convex continuous fuzzy subset of the real line whose membership function $\mu_{\tilde{\mathbf{P}}}\left(P_{1}\right)$ is defined by

1- A continuous mapping from $R$ to the closed interval $[0,1]$

$2-\mu_{\tilde{p}}\left(P_{i}\right)=0$ for all $p \in\left(-\infty, P_{1}\right]$

3- Stricyly increasing on $\left[P_{1}, P_{2}\right]$

4- $\mu_{\tilde{\mathbf{P}}}\left(P_{i}\right)=1$ for all $p \in\left[P_{2}, P_{3}\right]$

5- Strictly decreasing on $\left[P_{3}, P_{4}\right]$

6- $\mu_{\widetilde{\mathrm{P}}}\left(P_{\mathrm{i}}\right)=0$ for all $p \in\left[\mathrm{P}_{1}, \infty\right)$

figure (2.1) illustrate the graph of the possible shape of membership function of a fuzzy nuber $\widetilde{P}$

$\left(\mathbf{P}_{1}\right)$

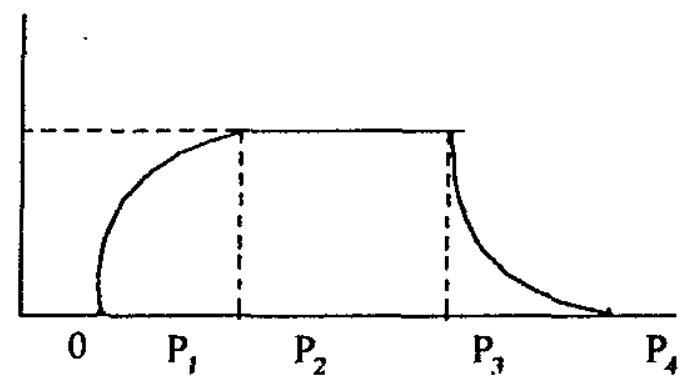

Figure (2.1) membersiungp function of fuzzy number $\widetilde{\mathrm{P}}$ 
Beflnition (2.2): ( $\alpha$ - cut)

The $\alpha$ - level set ( $\alpha$ - cut) of the fuzzy numbers $\widetilde{c}_{1}$ is defined as the ordinary set $L_{\mu}(\widetilde{c})$ for which the degree of their membership function exceeds the level $\alpha$ where

$\mathrm{L}_{\mu}(\widetilde{\mathrm{c}})=\left\{\mathrm{c} \mid \mu \widetilde{\mathrm{C}}_{1}\left(\mathrm{C}_{i}\right) \geq \alpha\right\}$

\section{3- Deterministic Form for (FMLP) Problem}

In this neclion the determinstic form for the fuzzy mitilevel linear mltilevel linear programming problem ( $\alpha$ - FMLP) is detmined and then a technique for determining its corresponding solutions also prroposed as:

For the first level (first DM).

$\alpha-$ FMLP (1):

$\max _{1} f_{1}\left(x_{1}, x_{2}, \ldots, x_{n}\right)=c_{1}^{(1)} x_{1}+c_{2}^{(1)} x_{2}+\ldots \ldots+c_{n}{ }^{(1)} x_{n}$ 、

where $\quad\left(x_{2}, x_{3}, \ldots, x_{n}\right)$ solves

$\max _{\mathrm{X}_{2}} \mathrm{f}_{2}\left(\mathrm{x}_{1}, \mathrm{x}_{2}, \ldots, \mathrm{x}_{n}\right)=\mathrm{c}^{(2)} \quad \cdots \mathrm{x}_{2}+\ldots \ldots+\mathrm{c}_{n}{ }^{(2)} \mathrm{x}_{n}$ '

where $\quad x_{n-1}$ solves

$\max _{n-1} f_{n-1}\left(x_{1}, x_{2}, \ldots, x_{n}\right)=c_{1}^{(n-1)} x_{1}+c_{2}{ }^{(n-1)} x_{2}+\ldots \ldots+c_{n}{ }^{(n-1)} x_{n}$ '

where $\quad x_{n} \quad$ solves

$\max _{n} f_{n}\left(x_{1}, x_{2}, \ldots, x_{n}\right)=c_{1}^{(n)} x_{1}+c_{2}^{(n)} x_{2}+\ldots . .+c_{n}^{(n)} x_{n}$

subject to

$\mathrm{G}_{\mathrm{i}}\left(\mathrm{x}_{1}, \mathrm{x}_{2}, \ldots, \mathrm{x}_{11}\right) \leq \mathrm{b}$,

$s_{1} \leq c_{i}^{(1)} \leq T_{i}$

$x_{1}, x_{2}, \ldots, x_{n} \geq 0$

For the second level (second DM)

$\alpha-\operatorname{EMLP}(2):$

$\max _{\mathrm{x}_{2}} \mathrm{f}_{2}\left(\mathrm{x}_{1}^{0}, \mathrm{x}_{2}, \ldots, \mathrm{x}_{\mathrm{n}}\right)=\mathrm{c}_{1}^{(2)} \mathrm{x}_{1}^{0}+\mathrm{c}_{2}^{(2)} \mathrm{x}_{2}+\ldots \ldots+\mathrm{c}_{\mathrm{n}}^{(2)} \mathrm{x}_{\mathrm{n}}$ where $\quad x_{n-1}$ solves 
$\max _{n-1} f_{n-1}\left(x_{1}^{0}, x_{2}, \ldots, x_{n}\right)=c_{1}^{(n-1)} x_{1}^{0}+c_{2}^{(n-1)} x_{2}+\ldots .+c_{n}^{(n-1)} x_{n}$ where $x_{n} \quad$ solves

$\max _{n} f_{n}\left(x_{1}^{0}, x_{2}, \ldots, x_{n}\right)=c_{1}^{(n)} x_{1}^{0}+c_{2}^{(n)} x_{2}+\ldots .+c_{n}{ }^{(n)} x_{n}$ subject to

$G_{i}\left(x_{1}^{0}, x_{2}, \ldots, x_{n}\right) \leq b$,

$S_{i} \leq C_{i}^{(2)} \leq T_{i}$

$x_{1}{ }^{\prime \prime}, x_{2}, \ldots, x_{n} \geq 0$

and so on to the nth decision maker (level).

Then the Kulw- Tucker conditions for the above multilevel mathematical programming model at the point $\left(\bar{X}_{1}, \bar{X}_{2}, \ldots ., \bar{X}_{n}\right)$ and the coefficient $\left(\overline{\mathrm{C}}_{1}, \overline{\mathrm{C}}_{2}, \ldots, \overline{\mathrm{C}}_{\mathrm{n}}\right)$ can be determined as:

$$
\frac{\partial \mathrm{L}_{1}(\overline{\mathrm{x}}, \overline{\mathrm{c}}, \lambda, \phi, \psi, \mu)}{\partial \mathrm{x}_{1}}=0 \text {, }
$$

$\frac{\partial L_{1}(\overline{\mathrm{x}}, \overline{\mathrm{x}}, \lambda, \phi, \psi, \mu)}{\partial \mathrm{c}_{1}^{(1)}}=0$

$\frac{\partial \mathrm{L}_{1}(\overline{\mathrm{x}}, \overline{\mathrm{c}}, \lambda, \phi, \psi, \mu)}{\partial \mathrm{x}_{2}}=0$

$\frac{\partial \mathrm{L}_{1}(\overline{\mathrm{x}}, \overline{\mathrm{c}}, \lambda, \phi, \psi, \mu)}{\partial \mathrm{c}_{1}^{(2)}}=0$,

$\frac{\partial \mathrm{L}_{n}(\overline{\mathrm{x}}, \overline{\mathrm{c}}, \lambda, \phi, \psi, \mu)}{\partial \mathrm{x}_{\mathrm{n}}}=0$

$\frac{\partial L_{n}(\overline{\mathrm{x}}, \overline{\mathrm{c}}, \lambda, \phi, \psi, \mu)}{\partial \mathrm{c}_{\mathrm{i}}^{(\mathrm{n})}}=0$ 


$$
\begin{aligned}
& \left(\sum_{i=1}^{n} G_{i}\left(X_{1}\right)-b_{1}\right) \leq 0 \\
& \lambda_{1}^{T}\left(\sum_{i=1}^{n} G_{i}\left(X_{1}\right)-b_{1}\right)=0 \\
& \phi_{i}^{T}\left(S_{1}^{(1)}-c_{1}^{(1)}\right)=0, \quad i=1,2, \ldots ., n, \\
& \psi_{i}^{T}\left(c_{1}^{(1)}-T_{i}^{(1)}\right)=0, \quad i=1,2, \ldots ., n, \\
& \phi_{i}^{T}\left(S_{1}^{(2)}-c_{1}^{(2)}\right)=0, \quad i=1,2, \ldots ., n, \\
& \psi_{i}^{T}\left(c_{1}^{(2)}-T_{i}^{(2)}\right)=0, \quad i=1,2, \ldots ., n, \\
& \phi_{i}^{T}\left(S_{i}^{(n)}-c_{i}^{(n)}\right) \quad=0, \quad i=1,2, \ldots ., n, \\
& \psi_{i}^{T}\left(c_{i}^{(n)}-T_{i}^{(n)}\right) \quad=0, \quad i=1,2, \ldots ., n, \\
& \mu_{i}^{T} x_{i}=0 x_{i} \geq 0, i=1,2, \ldots ., n,
\end{aligned}
$$

where

$\mathrm{L}_{\mathrm{i}}(\mathrm{x}, \mathrm{c}, \lambda, \phi, \psi, \mu)=$

$$
\begin{aligned}
& f_{i}\left(x_{1}, x_{2}, \ldots, x_{n}\right)+\sum_{i=1}^{n} \lambda_{i}^{T}\left(\sum_{i=1}^{n} G_{i}\left(x_{i}\right)-b_{1}\right)+\sum_{j 1}^{n} \sum_{i=1}^{n} \phi_{i}^{(j)} \\
& T\left(S_{1}^{(j)}-C_{i}^{(j)}\right)+\sum_{j=1}^{n} \sum_{i=1}^{n} \psi_{i}^{(j)} T\left(c_{i}^{(j)}-T_{i}^{(j)}\right)+\mu_{i}^{T} x_{i}
\end{aligned}
$$

\section{4- An illustrative Example}

Consider the following fuzzy Multilevel programming problem, the example formulate in bilevel programming form to clarify the idea; so it takes the form'

FMLP:

$$
\max _{x} f_{1}(x, y)=c_{1}^{(1)} x+c_{2}^{(1)} y
$$


where y solves

$$
\max _{y} f_{2}(x, y)=c_{1}^{(2)} x+c_{2}^{(2)} y \text {, }
$$

subject to

$$
\begin{aligned}
& x+y \geq 4, \\
& x+2 y \geq 6, \\
& S_{1}^{1} \leq C_{1}^{(1)} \leq T_{1}^{1}, \\
& S_{1}^{1} \leq C_{1}^{(1)} \leq T_{1}^{1}, \\
& S_{1}^{2} \leq C_{1}^{(2)} \leq T_{1}^{2}, \\
& S_{2}^{2} \leq C_{2}^{(2)} \leq T_{2}^{2}, \\
& x, y \geq 0
\end{aligned}
$$

and the corresponding lagrangian functions [3] for $\alpha$ - EMLP is

$$
\begin{aligned}
& \mathrm{L}_{j}(\mathrm{x}, \mathrm{y}, \mathrm{c}, \lambda)= \\
& \left(\mathrm{c}_{1}^{(j)} \mathrm{x}+\mathrm{c}_{2}^{(\mathrm{j})} \mathrm{y}\right)-\lambda_{1}^{\mathrm{j}}(\mathrm{x}+\mathrm{y}-4)-\lambda_{2}^{\mathrm{j}}(\mathrm{x}+2 \mathrm{y}-6)-\lambda_{3}^{\mathrm{j}}\left(\mathrm{T}_{1}^{1}-\mathrm{c}_{1}^{(1)}\right) \\
& -\lambda_{4}^{\mathrm{j}}\left(\mathrm{c}_{1}^{(1)}-\mathrm{S}_{1}^{1}\right)-\lambda_{6}^{\mathrm{j}}\left(\mathrm{c}_{2}^{(1)}-\mathrm{S}_{2}^{\mathrm{j}}\right)-\lambda_{7}^{\mathrm{j}}\left(\mathrm{T}_{1}^{2}-\mathrm{c}_{1}^{(2)}\right)-\lambda_{\mathrm{z}}^{\mathrm{j}}\left(\mathrm{c}_{1}^{(2)}-\mathrm{S}_{1}^{2}\right) \\
& -\lambda_{9}^{j}\left(\mathrm{~T}_{2}^{2}-\mathrm{c}_{2}^{(2)}\right)-\lambda_{10}^{\mathrm{j}}\left(\mathrm{c}_{2}^{(2)}-\mathrm{S}_{2}^{2}\right)-\lambda_{11}^{\mathrm{j}} \mathrm{x}-\lambda_{12}^{\mathrm{j}} \mathrm{y}, \mathrm{j}=1.2
\end{aligned}
$$

Then the corresponding Kuhn- Tucker are

$$
\begin{array}{ll}
\frac{\partial L_{j}}{\partial x}=c_{1}^{(j)}-\lambda_{1}^{i}-\lambda_{2}^{j}-\lambda_{11}^{j}=0, & \frac{\partial L_{j}}{\partial y}=c_{1}^{(j)}-\lambda_{1}^{j}-2 \lambda_{2}^{j}-\lambda_{12}^{j}=0, j=1,2, \\
\frac{\partial L_{1}}{\partial c_{1}^{(1)}}=x+\lambda_{3}^{1}-\lambda_{4}^{1}=0 & , \frac{\partial L_{1}}{\partial c_{2}^{(1)}}=y+\lambda_{5}^{1}-\lambda_{6}^{1}=0 \\
\frac{\partial L_{2}}{\partial c_{1}^{(2)}}=\lambda_{7}^{1}-\lambda_{8}^{2}=0 & , \frac{\partial L_{1}}{\partial c_{2}^{(2)}}=\lambda_{9}^{1}-\lambda_{10}^{1}=0 \\
\frac{\partial L_{2}^{\prime}}{\partial c_{1}^{(1)}}=\lambda_{3}^{2}-\lambda_{4}^{2}=0 & , \frac{\partial L_{2}}{\partial c_{2}^{(1)}}=\lambda_{5}^{2}-\lambda_{6}^{2}=0
\end{array}
$$




$$
\begin{aligned}
& \lambda_{1}^{j}(x+y-4)=0 \\
& , \quad \lambda_{2}^{j}(x+2 y-6)=0, j=1,2, \\
& \lambda_{3}^{i}\left(T_{1}^{1}-c_{1}^{(1)}\right)=0 \\
& \text {, } \lambda_{4}^{i}\left(c_{1}^{(1)}-S_{1}^{1}\right)=0, j=1,2 \text {, } \\
& \lambda_{5}^{i}\left(\mathrm{~T}_{2}^{i}-\mathrm{c}_{2}^{(j)}\right)=0 \\
& \text {, } \lambda_{6}^{i}\left(c_{2}^{(1)}-S_{2}^{\prime}\right)=0, j=1,2 \text {, } \\
& \lambda_{7}^{i}\left(\mathrm{~T}_{1}^{2}-\mathrm{c}_{1}^{(2)}\right)=0 \\
& \lambda_{8}^{i}\left(c_{1}^{(2)}-S_{1}^{2}\right)=0, j=1,2 \text {, } \\
& \lambda_{9}^{i}\left(\mathrm{~T}_{2}^{2}-\mathrm{c}_{2}^{(2)}\right)=0 \\
& \text {, } \lambda_{10}^{i} \cdot\left(c_{2}^{(2)}-S_{2}^{2}\right)=0, j=1,2 \text {, } \\
& \lambda_{11}^{j} \mathrm{x}=0 \\
& \text {, } \lambda_{12}^{j} \mathrm{y} \\
& =0, \mathrm{j}=1,2 \\
& (x+y-4) \geq 0 \\
& \text {, }(x+2 y-6) \geq 0 \text {, } \\
& \left(\mathrm{T}_{1}^{1}-\mathrm{c}_{1}^{(1)}\right)=0 \\
& \text {, }\left(c_{1}^{(1)}-S_{1}^{\prime}\right)=0 \text {, } \\
& \left(\mathrm{T}_{2}^{1}-\mathrm{c}_{2}^{(1)}\right)=0 \\
& \text {, }\left(\mathrm{T}_{2}^{2}-\mathrm{c}_{1}^{(2)}\right)=0 \text {, } \\
& \left(\mathrm{T}_{1}^{2}-\mathrm{c}_{1}^{(2)}\right)=0 \\
& \left(c_{1}^{(2)}-S_{1}^{2}\right)=0 \text {, } \\
& \left(c_{2}^{(2)}-S_{2}^{2}\right)=0 \\
& \left(-2-c_{2}^{(2)}\right)=0 \text {, } \\
& \text { and } x \geq 0, y \geq 0, \lambda_{k}^{j} \geq 0 \\
& , \quad j=1,2, \ldots, ., 12
\end{aligned}
$$

To obtain $\mathrm{c}_{1}^{(1)}, \mathrm{c}_{2}^{(1)}, \mathrm{c}_{1}^{(2)}$, and $\mathrm{c}_{2}^{(2)}$ Take the cut $\alpha=0.5$

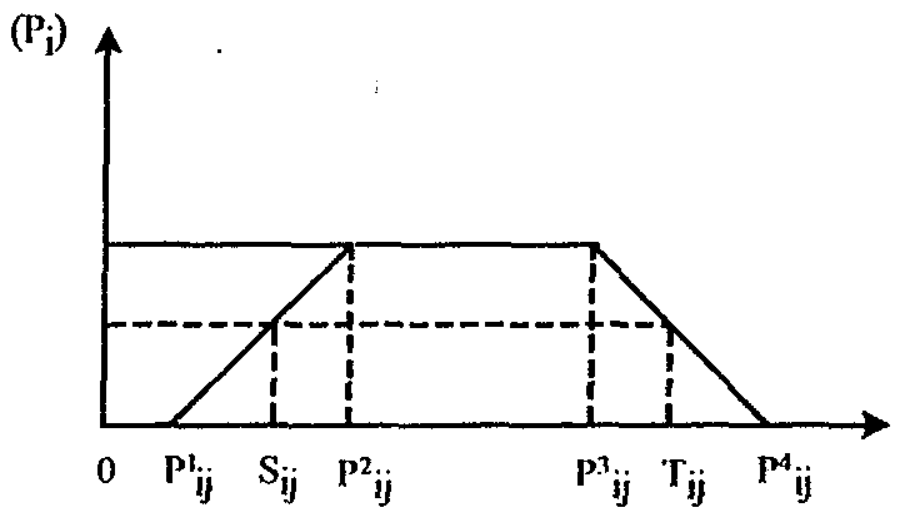




$$
\mu_{o i j}\left(\bar{c}_{i j}\right)= \begin{cases}0 & ,-\infty<c_{i j} \leq P_{i 1} \\ \frac{c_{i j}-P_{i 2}}{P_{i 2}-P_{i 1}} & , P_{i 2} \leq c_{i j} \leq P_{i 2} \\ 1 & , \\ \frac{c_{i j}-P_{i 4}}{P_{i 3}-P_{i 4}} & , P_{i j} \leq P_{i 3} \leq c_{i j} \leq P_{i 4} \\ 0 & , P_{i 4} \leq c_{i j} \leq \infty\end{cases}
$$

\begin{tabular}{|c|c|c|c|c|c|c|}
\hline$c_{j}^{i}$ & $P_{i j}^{1}$ & $P_{i j}^{2}$ & $P_{i j}^{3}$ & $P_{i j}^{4}$ & $S_{i j}$ & $T_{i j}$ \\
\hline$c_{1}^{1}$ & 3 & 5 & 6 & 8 & 6 & 7 \\
\hline$c_{2}^{1}$ & 2 & 4 & 6 & 8 & 5 & 7 \\
\hline$c_{2}^{2}$ & 4 & 6 & 8 & 10 & 7 & 9 \\
\hline$c_{2}^{2}$ & 3 & 5 & 6 & 10 & 6 & 8 \\
\hline
\end{tabular}

Now the two systen K-T (1), K-T(2) can be solve to obtain the value of the lagrangian multipliers and the solution set for the systems, where the parameters $c_{1}^{(1)}, c_{2}^{(1)}, c_{1}^{(2)}$, and $c_{2}^{(2)}$ can be obtained from the previous table.

\section{References}

1- I). Dubois \& H. Prade, Fuzzy sets and systems (Theory and applications), Academlc press, New York. (1980).

2- H. .J. Zimmermous, Fuzzy Set Theory and its applicalion, Kluwer-Nijhoff publishing. Boston, USA. (1988).

3- Mangasarian O., "Nonlinear Programming", McGraw-Hill, Inc., New York, London, 1964. 
4- M. Sakawa \& Yano, Interactive Decision Making For Mulliobjective Nonlinear Programming Problems with Fuzzy Parameters, Fuzzy set and system, 29 (1989), 315- 326.

5- M. S. Osman and A. Z. El-Bamma, Stability of multiobjective nonlinear programming problems with fuzzy parameters Mathematics and computers in simulation 35 (1993) 312- 326.

6- M. W. Carter, "The Indefinite 0-1 Quadratic problem" Discrete Applied Mathematics, 7 (1984), no., 1, 23-44.

7- O.Ben-Ayed, "Bilevel linear Programming". Computer Ops Res. Vol. 20, No. 5 pp. 485-501, 1993.

8- S. French, R. Hartley, L. Thomas and D. H. White, "Operational Research Techniques" Eward Arond, London, 1986. 


\section{البزمزفة الرياضية المتعددة المستويات الفازية}

محسد عبد العاطظى معاطى معدد مدرس بقسم الرياضيات والحاسب- كلية العلوم جامعة القاهرة (النيوم)

\section{ملخص البحث:}

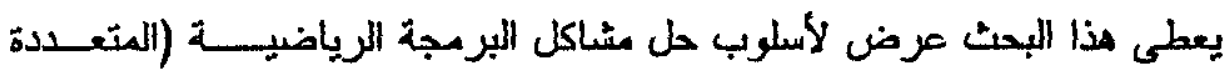

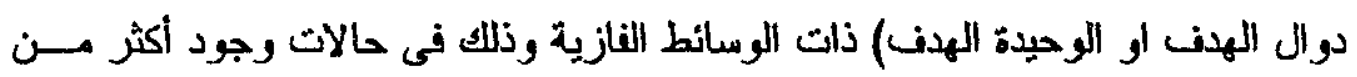
متذذ قرار فى تسلسل إدارى هرمى (عدم المركزية في إتخاذ القرار).

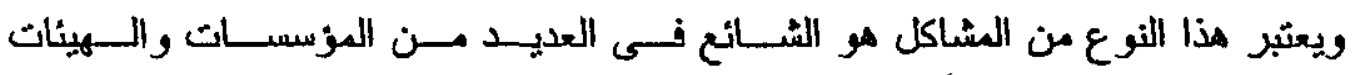

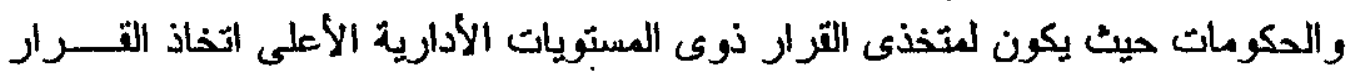
واضعيين فى الاعتبار ثرارات المعاونين لهم ذوى المتسويات الإدارية الألمل. 